\title{
Localized Hepatic Tuberculosis Presenting as Fever of Unknown Origin
}

\author{
Teresa Cristina Abreu Ferrari, \\ Carolina Mundim Couto, Tatiane Silva Vilaça and \\ Marcelo Antônio Pascoal Xavier
}

\author{
Federal University of Minas Gerais, Belo Horizonte, MG, Brazil
}

\begin{abstract}
Localized hepatic tuberculosis is a rare clinical form of tuberculosis infection; it has signs and symptoms related only to hepatic injury, with minimal or no extrahepatic involvement. It frequently presents as a non-specific syndrome, with systemic manifestations, which can sometimes result in a diagnostic dilemma. A high index of suspicion is required and a definitive diagnosis can be very difficult. We report a case of localized hepatic tuberculosis that presented as fever of unknown origin. Key Words: Tuberculosis, fever of unknown origin, granulomatous hepatitis.
\end{abstract}

Liver involvement may occur in primary and secondary forms of tuberculosis; it is particularly frequent in patients with disseminated miliary tuberculosis [1]. The local form of hepatic tuberculosis is much less common [2,3]. Its clinical presentation can be variable, and it is usually non-specific. Signs and symptoms include fever, hepatomegaly, night sweats, weight loss, malaise, anorexia, and abdominal pain. Occasionally, the illness can present as fever of unknown origin (FUO) [3]. Tuberculosis, mainly cases with extrapulmonary localization, remains an important cause of FUO, and hepatic tuberculosis should be considered in the differential diagnosis of this condition, especially if there is hepatomegaly of unknown origin $[3,4]$. Laboratory parameters and imaging methods in the local form of hepatic tuberculosis are frequently abnormal, but non-specific. Definitive diagnosis of this condition can be very difficult; it relies on histological and/or bacteriological findings of the liver tissue obtained by biopsy [2,3]. Sometimes, clinical diagnosis of tuberculosis is only confirmed after complete recovery with specific treatment. A good outcome is generally expected with early antituberculous therapy $[3,5]$. We present a case of this rare form of tuberculosis that presented as FUO, and we reinforce the importance of considering this diagnosis in any case of unexplained hepatomegaly, hepatosplenomegaly or FUO.

\section{Case report}

A 17-year-old man was admitted to the University Hospital, Federal University of Minas Gerais, with daily fever bouts (up to $\left.39.5^{\circ} \mathrm{C}\right)$, anorexia and weight loss $(6 \mathrm{~kg})$ during six months. $\mathrm{He}$ also complained of a mild dull ache in the right upper abdomen during the previous five weeks. Physical examination

Received on 22 May 2006; revised 21 September 2006.

Address for correspondence: Dr. Teresa Cristina Abreu Ferrari. Federal University of Minas Gerais, Faculty of Medicine, Department of Internal Medicine. Av. Professor Alfredo Balena, 190. Zip code: 30130-100, Belo Horizonte, MG, Brazil. Phone: +55 3132489746 , Fax: +55 313248 9664. E-mail: tferrari@ medicina.ufmg.br

The Brazilian Journal of Infectious Diseases 2006;10(5):364-367. (C) 2006 by The Brazilian Journal of Infectious Diseases and Contexto Publishing. All rights reserved. was unremarkable, except for a poor general condition and a tenderness associated with hepatosplenomegaly. Previous evaluation at another institution was inconclusive. Laboratory investigations showed hemoglobin, $9.9 \mathrm{~g} / \mathrm{dL}$; white blood cell count, 32,100 cells $/ \mathrm{mm}^{3}$, with a shift to the left; platelet cell

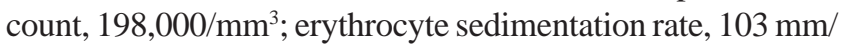
h; prothrombin time, $58 \%$; bilirrubin, $2.2 \mathrm{mg} / \mathrm{dL}$ (direct fraction, $1.2 \mathrm{mg} / \mathrm{dL}$ ); albumin, $3.1 \mathrm{~g} / \mathrm{dL}$; globulin, $4.0 \mathrm{~g} / \mathrm{dL}$; alanine aminotransferase (ALT), 126 U/L (normal, 7 - 30 U/L); aspartate aminotransferase (AST), $133 \mathrm{U} / \mathrm{L}$ (normal, 11 - $37 \mathrm{U} / \mathrm{L}$ ); and alkaline phosphatase, $346 \mathrm{U} / \mathrm{L}$ (normal, 15 - $69 \mathrm{U} / \mathrm{L}$ ). Renal function tests and urinalysis were within normal ranges. Serology for toxoplasmosis, brucellosis, hepatitis and leishmaniasis were negative. Blood cultures were negative, but the patient had recently taken antibiotics. Bone marrow examination showed no fungi, mycobacteria, parasites or atypical cells. Chest x-ray was normal. Abdominal ultrasonography demonstrated moderate increase of the liver and spleen. Rectal biopsy revealed viable eggs of Schistosoma mansoni. Liver biopsy was not performed at that time because the patient refused to be submitted to the procedure. Because of suspicion of Salmonella-S. mansoni association, the patient was treated with praziquantel and cloranfenicol, with no response. Since the symptoms persisted, he agreed to the proposed hepatic biopsy, which was guided by laparoscopy. Blood counts and liver function tests remained unaltered. During the laparoscopy, multiple nodules $0.2-0.6 \mathrm{~cm}$ in diameter could be seen on the liver surface, along with altered thickness of hepatic capsule, perihepatitis, and adherences between the epiplon and the anterior abdominal wall. Histophatological examination of the hepatic biopsy, stained by haematoxylin-eosin, Gomori and Ziehl-Neelsen, showed a diffuse and predominantly-mononuclear cell infiltrate with caseating necrosis foci that were surrounded by epithelioid cells, suggestive of granulomatous hepatic inflammation of tuberculous etiology. No fungi or acid-fast bacilli were found. The chest x-ray remained normal, and the tuberculin test was strongly positive. Antituberculous therapy, including isoniazid, rifampin and pyrazinamide, was initiated. Within a few days, the fever disappeared and a steady progressive improvement of the general condition of the patient was observed. Thirty days after the beginning of treatment, there 
was no hepatosplenomegaly, laboratory tests were normal, and the patient gained weight $(3.5 \mathrm{~kg})$. These drugs were maintained for six months. One year after the completion of treatment the patient remained completely asymptomatic.

\section{Discussion}

Fever of unknown origin constitutes one of the greatest challenges of clinical practice. It has been categorized into four variants: classic, neutropenic, HIV associated, and nosocomial. The definition of the classic type is a febrile disease during more than three weeks, with fever above $38.3^{\circ} \mathrm{C}$ on several occasions, which remains undiagnosed after three days of investigation in the hospital or after three outpatient visits [6-8].

Multiple etiologies should be considered when FUO is present; they can be grouped into the following categories: infection, neoplasia, collagenosis, miscellaneous, and undiagnosed causes. However, infection is the most common cause and accounts for approximately $20 \%$ to $40 \%$ of cases. Tuberculosis, mainly of extrapulmonary localization, remains an important cause of FUO $[4,9,10]$. For example, in a prospective study of 34 adult patients with FUO, tuberculosis, in varied clinical forms, was the most frequent disorder ( $40 \%$ of the patients assigned to the infectious diseases group and $17.6 \%$ of all cases in the series) [4]. In a more recent series, tuberculosis also constituted $40 \%$ of the patients with infectious diseases as a cause of FUO [11]. Approximately $36 \%$ of the cases in reports of granulomatous hepatitis presented as FUO, and hepatic granulomas have been associated with a wide variety of diseases of diverse etiologies, with 5-36\% of these remaining undiagnosed [10]. Hepatobiliary disorders can account for up to $25 \%$ of the causes of FUO [12]. The most common etiologies of these are infectious or inflammatory processes (hepatitis, cholangitis, cholecystitis), and neoplastic disorders [13].

Localized hepatic tuberculosis is a distinct clinical form of tuberculosis, with signs and symptoms related only to the hepatic injury, with minimal or no extrahepatic involvement. The clinical characterization and the nomenclature of isolated hepatic tuberculosis are not clearly defined; this disorder is referred to as atypical tuberculosis of the liver, tuberculous hepatitis, hepatic tuberculosis, hepatobiliary tuberculosis and localized or local hepatic tuberculosis. Hepatic tuberculosis constitutes less than $1 \%$ of all cases of this infection [14]. Liver involvement may occur in the primary and secondary forms of tuberculosis and is particularly frequent in patients with disseminated miliary tuberculosis. In autopsy series of disseminated tuberculosis, liver involvement was found in $80-100 \%$ of the cases [1]. On the other hand, the local form of hepatic tuberculosis, with minimal or no extrahepatic manifestations, is much less common [2,3]. Kok et al. [15] reported that hepatic tuberculosis was isolated in $0.3 \%$ of 1,678 new cases of tuberculosis.
It is believed that pathogenesis of these two forms of hepatic tuberculosis is different. Hematogenous dissemination of the bacteria seems to be the route by which the bacilli reach the liver in miliary hepatic tuberculosis; on the other hand, in local hepatic tuberculosis, the tubercle bacillus probably reaches the liver from the intestine via the portal vein. The possibility of such mechanisms is reinforced by the histopathological findings; in miliary tuberculosis, the granulomas are nearly always situated inside the lobules and in the local hepatic form they are mainly in the portal regions [3].

Patients with hepatic tuberculosis have variable clinical presentations and no consistent clinical and biochemical findings, which makes diagnosis difficult. The presenting symptoms are usually non specific and are mainly constitutional in nature; they include fever, night sweats, malaise, anorexia, weight loss, and abdominal pain $[14,15]$. In general, the clue to the diagnosis of hepatic involvement is the finding of tuberculosis elsewhere. When such evidence is lacking, a correct diagnosis can be extremely difficult, as in our case study, which presented as FUO. Disturbance of bowel habit may be present, and diarrhea was about twice as common as constipation in another study [3]. Abdominal tenderness in the epigastrium or right upper quadrant is a common manifestation [15]. Hepatomegaly is observed in most cases and has been frequently associated with splenomegaly [1]; however, splenic involvement is more common and extensive in the miliary form [16]. Jaundice may occur and is attributed mainly to the direct destruction of the liver parenchyma by tuberculosis [15], but obstructive processes may also be present [16].

The most frequent clinical-laboratory findings in tuberculosis of the liver are hepatomegaly ( $90 \%)$, elevated serum alkaline phosphatase levels $(\sim 80 \%)$, fever $(\sim 70 \%)$, weight loss ( $60 \%)$, and abdominal pain ( $55 \%)$ [3,5]; all of them were present in our case. A moderate or marked increase in the serum levels of alkaline phosphatase, along with normal or mildly increased serum bilirrubin, is considered suggestive of hepatic tuberculosis; however, these findings are not specific and may occur in other conditions, such as metastatic carcinoma, liver abscess, echinococcosis, amyloidosis, granulomatous diseases of varying etiologies, and active cirrhosis [3]. This pattern of biochemical alterations was also observed in our patient. Low serum-albumin levels and hyperglobulinaemia have also been described as suggestive of hepatic tuberculosis $[2,3]$. The aminotransferases can be moderately elevated or normal, and gamma glutamyltranspeptidase levels are sometimes markedly raised. Abnormal prothrombin time has been a common finding in some series [3]. Non-specific laboratory alterations, such as anemia and leukocytosis can be found. Sometimes there is pancytopenia. Increased erythrocyte sedimentation rates are common $[5,17]$. Most of these laboratory abnormalities were observed in our case study. 
Local and miliary forms of hepatic tuberculosis have similar biochemical presentations, but the local form is associated with more severe hepatocytic damage (higher serum ALT levels), and the miliary form is more wasting (lower serum albumin levels) [2]. The strong impairment of the general condition of our patient was probably due to the long duration of the disease condition.

Histological findings may include a wide variety of hepatic lesions. In a clinical review of 96 cases of patients with a predominantly hepatic presentation of tuberculosis, the findings were: granulomas (95.8\%), caseation ( $83.3 \%$ ), fatty changes (42\%), portal fibrosis (20\%), and acid-fast bacilli in association with granulomas (9\%). A mononuclear cell infiltrate was also common [5]. In general, tubercle bacilli are rarely encountered and the finding of caseation in the hepatic tuberculous granulomas, although variable, is not very frequent [2,17-19]. Bacteria are cultivated from the liver in only $0-10 \%$ of the cases, with the highest yield coming from granulomas with caseating necrosis [10]. Certain distinctive features can identify tuberculous granulomas in the liver; these include acid-fast bacilli within the lesion, caseating necrosis with destruction of the reticulin framework, irregular contours with a particularly dense cuff of lymphocytes surrounding the lesion, and few lesions, with a tendency to coalesce [10]. However, the etiology of hepatic granulomas can seldom be established by histological appearance alone. Some authors suggest that, in a consistent clinical picture, which is frequently somewhat non-specific, the finding of granulomas, especially with caseating necrosis, constitutes histopathological evidence of tuberculosis, unless proven otherwise $[2,20]$.

According to a review of several series of granulomatous hepatitis, tuberculosis is the second-most-common single cause of hepatic granulomas (10\%-53\% of the cases), and it is by far the main cause of granulomatous hepatitis among infectious diseases. The most frequent identified cause was sarcoidosis, but these results must be cautiously interpreted, because they are dependent on a number of variables, including types of populations studied, geographic locations, and special interests of the investigators [10]. For example, Mir-Madjlessi et al. [21] focused on noncaseating granulomas and found a higher incidence of granulomas of unknown etiology.

Our patient also suffered from schistosomiasis; however, this infection probably had little or no effect on his clinical picture. The histological appearance of the liver in this case was not suggestive of schistosomiasis as a cause of granulomatous hepatitis. Schistosomal hepatic granulomas appear as aggregates of lymphocytes, macrophages and eosinophils, and the parasite eggs or their remnants are found within the granulomas [10].

A high degree of suspicion is required for diagnosing localized hepatic tuberculosis, and the definite diagnosis relies on histological and/or bacteriological evidence of infection
[15]. Histopathological examination of liver tissue obtained by biopsy is the most reliable diagnostic method [2,3]. Imaging methods are of little value, because the findings are nonspecific. Ultrasonography, computerized tomography, and magnetic resonance imaging are very sensitive for the detection of hepatosplenic nodules, but differential diagnosis from other conditions, such as metastases, fungal abscesses and lymphomas, is difficult [14]. The tuberculin skin test is of little value as a diagnostic method. Other conditions can be associated with a positive reaction, and this test can be negative in patients with tuberculosis $[10,17]$. Sometimes, clinical diagnosis of tuberculosis is confirmed only after complete recovery due to antituberculous therapy. Some authors suggest that, whenever there is a lack of etiological diagnosis of a granulomatous hepatitis, patients should be considered for an empirical trial with antituberculous drugs, especially if there is clinical deterioration, particularly in areas where tuberculosis is endemic $[10,17,19]$.

Tuberculous hepatitis is treated according to standard drug regimens. Response to treatment is less satisfactory in acutely-ill patients and in those younger than 20 years, as well as in patients with coagulopathy, high caseation scores, and those with predisposing factors, such as steroid treatment, chronic renal failure, diabetes mellitus and systemic lupus erythematosus. The success of treatment depends on early recognition of the disease, which can be very difficult, because of non-specific presentation in the great majority of cases, and because hepatic tuberculosis is easily confused with other liver diseases [5].

In conclusion, tuberculosis of the liver should be considered in any case of unexplained hepatomegaly, hepatosplenomegaly or FUO; and, in suspicious cases, a liver biopsy should be performed without delay, since this condition responds well to early antituberculous therapy.

\section{References}

1. Tritou I., Prassopoulos P., Daskalogiannaki M., et al. Miliary hepatic tuberculosis not associated with splenic or lung involvement. Acta Radiol 2000;41:478-81.

2. Chien R.N., Lin P.Y., Liaw Y.F. Hepatic tuberculosis: comparison of miliary and local form. Infection 1995;23:5-12.

3. Hersch C. Tuberculosis of the liver: a study of 200 cases. S Afr Med 1964;38:857-63.

4. Ferrari T.C.A., Pedroso E.R.P. Fever of unknown origin (FUO): a prospective study of 34 cases and a literature review. Rev Med Minas Gerais 1998;8:47-52.

5. Essop A.R., Posen J.A., Hodkinson J.H., Segal I. Tuberculous hepatitis: a clinic review of 96 cases. Q J Med 1984;53:465-77.

6. Petersdorf, R.G., Beeson, P.B. Fever of unexplained origin: report on 100 cases. Medicine 1961;40:1-30.

7. Petersdorf, R.G. Fever of unknown origin: an old friend revisited. Arch Intern Med 1992;152:21-6.

8. Konecny P., Davidson R.N. Pyrexia of unknown origin in the 1990s: time to redefine. Br J Hosp Med 1996;56:21-32. 
9. Knockaert D.C., Vanneste L.J., Vanneste S.B., Bobbaers H.J. Fever of unknown origin in the 1980s: an update of the diagnostic spectrum. Arch Intern Med 1992;152:51-5.

10. Harrington P.T., Gutiérrez J.J., Ramirez-Ronda C.H., QuiñonesSoto R., Bermúdez R.H., Chaffey J. Granulomatous hepatitis. Rev Infect Dis 1982;4:638-55.

11. Oropeza D.L.,Vásquez M.V., Bedoya P.A. Hepatitis granulomatosa tuberculosa como causa de fiebre de origen desconocido. Rev Gastroenterol Peru 2002;22:324-9.

12. Salama H.M., Abdel-Wahab M.F., Farid Z. Hepatobiliary disorders presenting as fever of unknown origin in Cairo, Egypt: the role of diagnostic ultrasonography. J Trop Med Hyg 1988;91:147-9.

13. Saidi R.F., ReMine S.G., Jacobs M.J. Intrahepatic cholangiocarcinoma masked as fever of unknown origin. J Gastrointest Surg 2004;8:217-9.

14. Mert A., Ozaras R., Tabak F., et al. Localized hepatic tuberculosis. Eur J Intern Med 2003;14:511-2.
15. Kok K.Y.Y., Yapp S.K.S. Isolated hepatic tuberculosis: report of five cases and review of the literature. J Hepatobiliary Pancreat Surg 1999;6:195-8.

16. Hickey N., McNulty J.G., Osborne H., Finucane J. Acute hepatobiliary tuberculosis: a report of two cases and a review of the literature. Eur Radiol 1999;9:886-9.

17. Palmer K.R., Patil D.H., Basran G.S., et al. Abdominal tuberculosis in urbain Britain - a common disease. Gut 1985;26:1296-305.

18. McCluggage W.G., Sloan J.M. Hepatic granulomas in Northern Ireland: a thirteen year review. Histopathology 1994;25:219-28.

19. Sabharwal B.D., Malhotra N., Garg R., Malhotra V. Granulomatous hepatitis: a retrospective review. Indian J Pathol Microbiol 1995;38:413-16.

20. Alvarez S.Z., Carpio R. Hepatobiliary tuberculosis. Dig Dis Sci 1983;28:193-200.

21. Mir-Madjlessi S.H., Farmer R.G., Hawk W.A. Granulomatous hepatitis: a review of 50 cases. Am J Gastroenterol 1973;60:122-34. 